\title{
Collective strong coupling in multimode cavity QED
}

\author{
A. Wickenbrock, ${ }^{1}$ M. Hemmerling, ${ }^{2}$ G. R. M. Robb, ${ }^{2}$ C. Emary, ${ }^{3}$ and F. Renzoni ${ }^{1}$ \\ ${ }^{1}$ Department of Physics and Astronomy, University College London, Gower Street, London WC1E 6BT, England, United Kingdom \\ ${ }^{2}$ Scottish Universities Physics Alliance, Department of Physics, University of Strathclyde, 107 Rottenrow, Glasgow G4 ONG, \\ Scotland, United Kingdom \\ ${ }^{3}$ Institut für Theoretische Physik, Technische Universität Berlin, D-10623 Berlin, Germany \\ (Received 30 July 2012; revised manuscript received 11 January 2013; published 15 April 2013)
}

\begin{abstract}
We study an atom-cavity system in which the cavity has several degenerate transverse modes. Mode-resolved cavity transmission spectroscopy reveals well-resolved atom-cavity resonances for several cavity modes, a signature of collective strong coupling for the different modes. Furthermore, the experiment shows that the cavity modes are coupled via the atomic ensemble contained in the cavity. The experimental observations are supported by a detailed theoretical analysis. The work paves the way to the use of interacting degenerate modes in cavity-based quantum information processing, where qubits corresponding to different cavity modes interact via an atom shared by the two modes. Our results are also relevant to the experimental realization of quantum spin glasses with ultracold atoms.
\end{abstract}

DOI: 10.1103/PhysRevA.87.043817

PACS number(s): 42.50.Pq, 37.30.+i, 05.40.-a

\section{INTRODUCTION}

Cavity quantum electrodynamics (CQED) studies the interaction of atoms with a quantized light field enclosed in a cavity [1-3]. In the early days of CQED, the focus was on the study of fundamental processes in atom-light interaction and the exploration of the quantum-classical interface as determined by decoherence processes [4]. More recently, a wealth of applications taking advantage of the atom-cavity coupling have been identified, from cavity-based quantum computing [5-9] to cavity cooling of atoms and molecules [10-12]. Most of the experimental and theoretical work so far has been devoted to single mode cavities. For these cavities, the regime of strong coupling was observed with single atoms, with dilute atomic samples, and with Bose-Einstein condensates $[13,14]$. The inclusion of multiple cavity modes in the dynamics is predicted to lead to an increase of the atom-field coupling and to the enhancement of several cavity effects, as for example the atomic enhanced entanglement in optical cavities [15] or an increase in the capture range of cavity cooling [16]. Furthermore, this also opens up new schemes in quantum information processing using different cavity modes as qubits interacting via the atoms stored in the cavity [17] and is an important element in the implementation of quantum spin glasses with ultracold atoms [18]. Multimode CQED was realized with single atoms interacting with different polarization cavity modes [19]. However, in this case the number of modes taking part in the process was limited to two. In order to have a larger number of modes, a nearly confocal cavity characterized by a large number of degenerate modes can be used. The strong-coupling regime in such a cavity was reported in [20], but since the mode structure could not be resolved no multimode features were identified.

In this work we study the coupled atom-light dynamics in a pumped nearly confocal optical cavity containing cesium atoms. The near confocality leads to a large number of degenerate transverse modes, a distinguishing feature of our CQED experiment. The dynamics involves multiatom multimode collective states. Mode-resolved cavity transmission spectroscopy, as introduced in this work, allows us to study the coupling of the atoms to the individual modes, as well as the coupling between modes via the atoms. Our experiment reveals well-resolved atom-cavity resonances for several cavity modes, a signature of collective strong coupling for the different modes. We demonstrate the coupling, mediated by the atoms, between different degenerate cavity modes. This is the key element in the possible implementation of quantum computing in cavities using different modes as qubits [17]. A theoretical and numerical analysis identifies the mechanisms behind our observation and demonstrates the importance of the atomic distribution within the modes.

This work is organized as follows. In Sec. II we define our setup and describe the experimental results. In Sec. III we introduce the theoretical model and interpret our experimental results on atom mediated modal coupling both with analytic and numerical solutions. We then extend the theoretical analysis beyond the parameter space of our experiment and describe and interpret the general situation of a multipeaked transmission cavity profile. Finally, in Sec. IV conclusions are drawn.

\section{EXPERIMENT}

The details of our cavity setup were published previously [20]. We recall here the essential features and detail the additional measuring apparatus for the imaging of the cavity modes. The central element of the experiment is a linear, nearly confocal optical resonator of length $L=(11.996 \pm 0.003) \mathrm{cm}$, as sketched in Fig. 1. The relevant single-mode, singleatom frequencies are $(g, \kappa, \gamma)=2 \pi(0.12,0.8,2.6) \mathrm{MHz}$. The system is thus in the "bad cavity" regime $(g \ll \kappa<\gamma)$. The beam waist $w_{00}$ of the $\mathrm{TEM}_{00}$ cavity mode is calculated from the length and the mirror curvature of the cavity to be $(127 \pm 1) \mu \mathrm{m}$. The cavity transmission is collimated and then imaged with an intensified CCD camera. The resulting beam waist on the CCD chip (square pixel size: $6.45 \mu \mathrm{m}$ ) is determined to be approximately $805 \mu \mathrm{m}$ by optimizing the modal decomposition with a reduced set of modes to the empty cavity transmission coupled mostly into the 00 mode. This value is consistent with a beampath analysis 


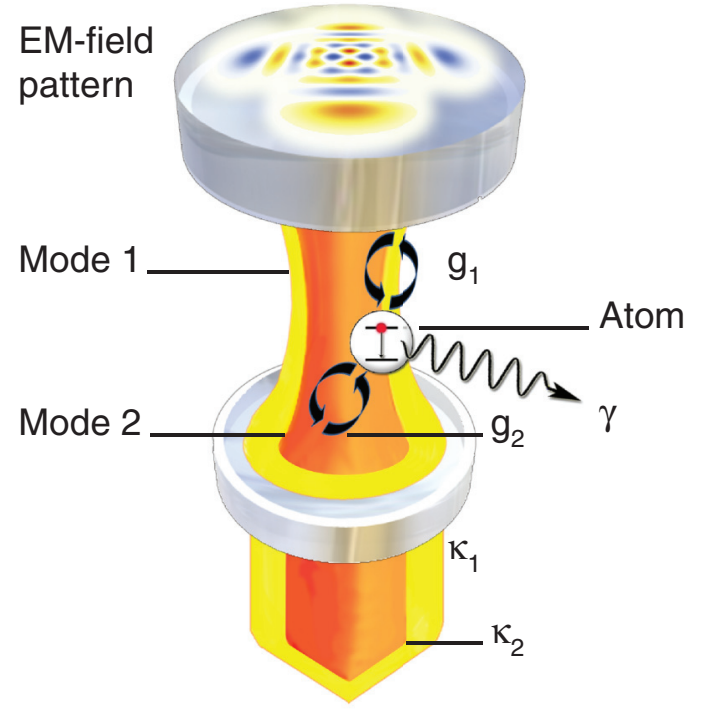

FIG. 1. (Color online) A two-level atom in an optical cavity interacting with different cavity modes depending on the spatially dependent couplings. Shown here are two higher-order transversal modes (modes 1 and 2) and one two-level atom exchange excitation with rates $g_{1}$ and $g_{2}$, respectively. The coherent process is damped by radiative coupling to the environment either via cavity decay of each mode through the mirrors (with rates $\kappa_{1}$ and $\kappa_{2}$ ) or atomic polarization decay (with rate $\gamma$ ).

using ray matrices and is kept fixed for all further image decompositions.

Using a model incorporating spherical aberration, astigmatism, and beam front curvature, the deviation from confocality $\Delta_{i}=L-R_{i}$, with $R_{i}$ being the radius of curvature of the mirrors along two orthogonal directions $x$ and $y$, is derived from transmission measurements of the empty cavity. It ranges from $\left(\Delta_{x}, \Delta_{y}\right)=(5.8 \pm 0.7,76 \pm 4)$ to $(22 \pm 1,94 \pm 3) \mu \mathrm{m}$ for maximum and minimum piezo elongation, respectively. From the resulting frequency difference between the modes we derive the numbers of quasidegenerate modes contained within a cavity linewidth to be 41 and 3 , respectively.

One experimental cycle involves loading a magneto-optical trap (MOT), placed into the center of the cavity, from the background gas of the chamber. The loading time is varied between 0.1 and $2 \mathrm{~s}$, resulting in different atom numbers between $8 \times 10^{4}$ and $2 \times 10^{6}$. Subsequently the MOT is switched off, and after a delay of $1 \mathrm{~ms}$ the cavity is pumped by a linearly polarized laser beam for $1 \mathrm{~ms}$, and its transmission is recorded with the CCD camera. The relevant parameters for the pumping field are the atomic and cavity-field detunings, defined as $\Delta_{A}=\omega_{P}-\omega_{A}$ and $\Delta_{C}=\omega_{P}-\omega_{C}$, respectively, where $\omega_{A}$ is the atomic transition angular frequency; $\omega_{C}$ is the cavity resonance frequency, which is the same for all modes, as they are assumed to be degenerate; and $\omega_{P}$ is the pump laser frequency. Before measuring the multimode splitting, the cavity is positioned on resonance with the $F_{g}=4 \rightarrow F_{e}=5 D_{2}$ line transition, i.e., $\omega_{A}=\omega_{C}$ and thus $\Delta_{A}=\Delta_{C}$. A typical experimental run starts with a probe laser detuning of $+100 \mathrm{MHz}$ from the $4-5$ transition. This is then successively reduced in steps of $2 \mathrm{MHz}$ until a final detuning of
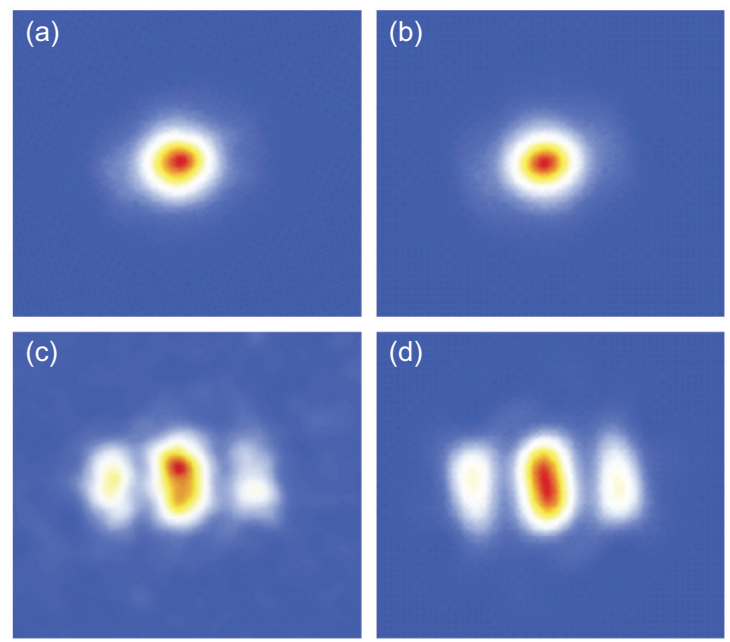

FIG. 2. (Color online) Typical images of the cavity transmission and their fits. (a) Transmission through the empty cavity dominated by the $\mathrm{TEM}_{00}$ mode and (b) corresponding fit. (c) Transmission through the atom-filled cavity with a strong $\mathrm{TEM}_{20}$ component and (d) corresponding fit.

$-100 \mathrm{MHz}$ is reached. For each frequency three transmission images are averaged.

In order to investigate the mode structure of the cavity field, the intensity profiles of the measured cavity transmission are fitted with

$$
F(x, y)=\left|\sum \alpha_{n m} E_{n m}(x, y)\right|^{2},
$$

where $E_{n m}(x, y)$ are Hermite-Gaussian functions and $\alpha_{n m}$ are the complex amplitudes. For a confocal cavity the mode spectrum reduces to two peaks per free spectral range. Our experiments investigate the peak containing the subset of higher-order transversal modes degenerate with the $\operatorname{TEM}_{q 00}$ mode. Thus, in our fit function Eq. (1) we only consider the terms $E_{n m}$ with $n+m$ even. The coupling into the cavity $\mathrm{TEM}_{00}$ mode was optimized by mode matching and the comparison of the transmission peak height of the odd with respect to the even modes. For the experiment, the odd modes were suppressed by at least a factor of 30 .

Typical output profiles and their fits can be seen in Fig. 2. A Lorentzian fit to the mode resolved empty cavity transmission (Fig. 3, black) reveals the modal weights of the pump beam to be $\left|\alpha_{00}\right|^{2}=0.879,\left|\alpha_{20}\right|^{2}=0.054,\left|\alpha_{02}\right|^{2}=0.065$, and $\left|\alpha_{11}\right|^{2}=0.002$. The relative weight changes when the cavity is loaded with atoms. These data show clear evidence of the multimode dynamics of the atom-cavity system.

A detailed quantitative study is reported in Fig. 3. The mode-resolved transmission, both for an empty cavity and for a cavity containing cesium atoms, is shown as a function of the atom-pump detuning. Normal-mode splitting is observed for several modes of the cavities. In fact, although we mostly pump the $\mathrm{TEM}_{00}$ mode, very well-resolved atom-cavity resonances are observed also for the modes $\mathrm{TEM}_{20}, \mathrm{TEM}_{02}$, and $\mathrm{TEM}_{11}$. In particular, transmission for the mode $\mathrm{TEM}_{11}$ shows no wellresolved central peak with an empty cavity; i.e., a negligible amount of light is coupled in this mode without the atoms. In contrast, with atoms present, this mode shows pronounced 


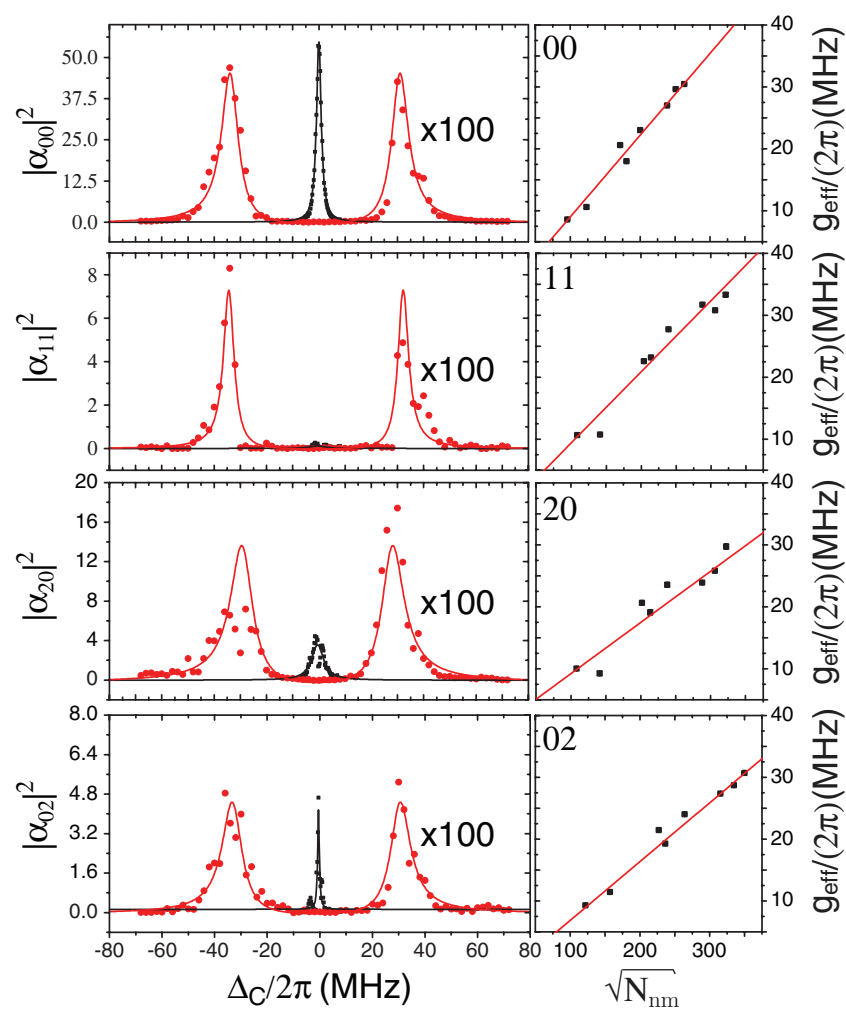

FIG. 3. (Color online) Left: Mode resolved intracavity photon number with atoms (red filled circles) and without atoms (black filled squares) in the cavity for different detunings $\Delta_{C} /(2 \pi)$. The number of atoms in the fundamental mode $\mathrm{TEM}_{00}$ of the cavity as derived from fluorescence images is $N_{00}=(100 \pm 8) \times 10^{3}$. The data points correspond to the absolute squared amplitude $\left|\alpha_{n m}\right|^{2}$ of the corresponding two-dimensional Hermite Gaussian mode function as derived from image fits with the function Eq. (1). The data were then fitted with a Lorentzian transmission function of an empty cavity and the theoretical transmission function of the coupled cavity-atom system, as from Eq. (2). All the displayed higher-order transversal modes exhibit the characteristic normal-mode splitting. We stress that for one of the data sets (the third figure from top, on the left) the deviation of the data from the fitting function is due to experimental limitations and does not constitute evidence of a further splitting. Right: The effective coupling for the $\mathrm{nm}$ mode plotted as a function of the square root of the number of atoms contained in that mode.

atom-cavity resonances, which demonstrates the mediation of coupling between different modes by the atoms.

We fit the data for each mode with the transmission function $T\left(\omega_{P}\right)[21]:$

$$
T\left(\omega_{P}\right)=T_{0}\left|\frac{\kappa_{\mathrm{eff}}\left[\gamma+i\left(\omega_{0}-\omega_{P}\right)\right]}{\left(\omega_{P}-\lambda_{+}\right)\left(\omega_{P}-\lambda_{+}\right)}\right|^{2},
$$

where $T_{0}$ is the maximum empty cavity transmission and $\lambda_{ \pm}=\left(\omega_{A} \pm \Omega_{0}\right)-i(\gamma+\kappa) / 2$ are the normal-mode eigenfrequencies of the coupled system, with $\Omega_{0}=\sqrt{g_{\text {eff }}^{2}-(\gamma-\kappa)^{2} / 4}$. The fit parameters are $T_{0}, \kappa_{\text {eff }}$, and $g_{\text {eff }}$. From our fits of the experimental data, we observed (see Fig. 3, right) that the effective coupling constant $g_{\text {eff }}$ for the $\mathrm{nm}$ mode scales as the square root of the number of atoms contained in that mode, as expected in the case of collective strong coupling.
Furthermore, the lines were observed to broaden with the atom number, with a width scaling as $N_{n m}^{1 / 2}$. We took this feature into account by including in the fitting function an effective $\kappa_{\text {eff }}$. As discussed below, such a broadening is a signature of the quasidegeneracy of the effective atom-cavity couplings.

\section{THEORY}

\section{A. Microscopic model}

We describe our system with an $N$-atom Tavis-Cummings model [22,23] extended to include $M$ cavity modes as well as an additional coherent driving term. The Hamiltonian of the atom-cavity system reads

$$
H=H_{S}+H_{D}=\left(H_{C}+H_{A}+V\right)+H_{D},
$$

where $H_{S}$ describes the "system," i.e., the cavity, the atoms, and their interactions, and $H_{D}$ describes the driving. In a frame rotating with the driving frequency, the cavity Hamiltonian reads ( $\hbar=1$ throughout)

$$
H_{C}=-\Delta_{C} \sum_{k=1}^{M} a_{k}^{\dagger} a_{k}
$$

where we have assumed that all $M$ modes are degenerate with detuning $\Delta_{C}$. The atomic Hamiltonian for $N$ two-level atoms reads

$$
H_{A}=-\frac{1}{2} \Delta_{A} \sum_{l=1}^{N} \sigma_{l}^{z},
$$

where $\Delta_{A}$ is the detuning of the atoms, assumed identical for all atoms. The interaction between the atoms and the cavity modes in the rotating wave approximation (RWA) reads

$$
V=\frac{1}{2} \sum_{k=1}^{M} \sum_{l=1}^{N}\left\{g_{k l} a_{k}^{\dagger} \sigma_{l}^{-}+g_{k l}^{*} a_{k} \sigma_{l}^{+}\right\} .
$$

Here, $a_{k}^{\dagger}$ and $a_{k}$ are the creation and the annihilation operators of $k$ th mode, where the index $k$ describes the complete set of modes previously labelled $n m, \sigma_{l}^{ \pm}$are the atomic raising and the lowering operators of $l$ th atom, and $g_{k l}$ is the coupling constant for the interaction between the $k$ th mode and the $l$ th atom, which depends on the atom via its position within the cavity.

We drive the system with a monochromatic laser, which we assume only couples to the cavity modes. In the RWA, we have

$$
H_{D}=\sum_{k=1}^{M} \eta_{k}\left(a_{k}^{\dagger}+a_{k}\right)
$$

with $\eta_{k}$ being the coupling strength of the laser to mode $k$. Dissipation due to cavity decay and spontaneous emission with homogeneous rates $\kappa$ and $\gamma$, respectively, is introduced in the standard quantum master equation approach.

\section{B. Weak excitation model}

We will consider that the effect of the driving term $H_{D}$ is weak enough that it introduces at most one excitation (atomic flip or cavity photon) into the system at any given time. This 
assumption allows us to restrict ourselves to the following set of relevant states:

$|0\rangle$ : no excitation (cavity vacuum, all atoms in ground state),

$\left|C_{k}\right\rangle$ : single-photon excitation in cavity mode $k$,

$\left|A_{l}\right\rangle$ : excitation of atom $l$.

In this basis, the component Hamiltonians of Eq. (3) (minus an offset) read

$$
\begin{gathered}
H_{C}=-\Delta_{C} \sum_{k=1}^{M}\left|C_{k}\right\rangle\left\langle C_{k}\right|, \\
H_{A}=-\Delta_{A} \sum_{l=1}^{N}\left|A_{l}\right\rangle\left\langle A_{l}\right|, \\
V=\frac{1}{2} \sum_{k=1}^{M} \sum_{l=1}^{N}\left(g_{k l}\left|C_{k}\right\rangle\left\langle A_{l}\left|+g_{k l}^{*}\right| A_{l}\right\rangle\left\langle C_{k}\right|\right), \\
H_{D}=\sum_{k=1}^{M} \eta_{k}\left(\left|C_{k}\right\rangle\langle 0|+| 0\rangle\left\langle C_{k}\right|\right) .
\end{gathered}
$$

\section{Singular value decomposition of the coupling matrix}

Let us write the coupling constants as the $M \times N$ matrix $G$ with elements $(G)_{k l}=g_{k l}$. The singular value decomposition (SVD) of $G$ reads

$$
G=U \Lambda W^{\dagger},
$$

where $U$ is an $M \times M$ unitary matrix, $W$ is an $N \times N$ unitary matrix, and $\Lambda$ is an $M \times N$ rectangular diagonal matrix. The nonzero diagonal elements of $\Lambda$ are the singular values of $G$, which we denote $\lambda_{j}$. The singular values are real, positive, and equal to the nonzero eigenvalues of the matrix $\Gamma \equiv \sqrt{G G^{\dagger}}$, and the number of singular values is equal to the rank $R_{\Gamma}$ of this matrix. Since, in our system, the number of modes is smaller than the number of atoms, $M<N$, the coupling matrix $G$ can have at most $M$ singular values, such that $R_{\Gamma} \leqslant M$.

Using this SVD we can rewrite the interaction as

$$
\begin{aligned}
V & =\frac{1}{2} \sum_{k=1}^{M} \sum_{l=1}^{N} \sum_{j=1}^{R_{\Gamma}} U_{k j} \lambda_{j}\left(W^{\dagger}\right)_{j l}\left|C_{k}\right\rangle\left\langle A_{l}\right|+\text { H.c. } \\
& =\frac{1}{2} \sum_{j=1}^{R_{\Gamma}}\left(\sum_{k=1}^{M} U_{k j}\left|C_{k}\right\rangle\right) \lambda_{j}\left(\sum_{l=1}^{N}\left(W^{\dagger}\right)_{j l}\left\langle A_{l}\right|\right)+\text { H.c. }
\end{aligned}
$$

We then define the collective cavity and atomic states:

$$
\begin{aligned}
& \left|\widetilde{C}_{j}\right\rangle=\sum_{k=1}^{M} U_{k j}\left|C_{k}\right\rangle \quad \text { (cavity), } \\
& \left|\widetilde{A}_{j}\right\rangle=\sum_{l=1}^{N} W_{l j}\left|A_{l}\right\rangle \quad \text { (atomic), }
\end{aligned}
$$

and we obtain

$$
V=\frac{1}{2} \sum_{j=1}^{R_{\Gamma}} \lambda_{j}\left(\left|\widetilde{C}_{j}\right\rangle\left\langle\widetilde{A}_{j}|+| \widetilde{A}_{j}\right\rangle\left\langle\widetilde{C}_{j}\right|\right) .
$$

In this new basis, the remaining parts of the system Hamiltonian read

$$
\begin{aligned}
& H_{C}=-\Delta_{C} \sum_{k=1}^{M}\left|\widetilde{C}_{k}\right\rangle\left\langle\widetilde{C}_{k}\right|, \\
& H_{A}=-\Delta_{A} \sum_{k=1}^{M}\left|\widetilde{A}_{k}\right\rangle\left\langle\widetilde{A}_{k}\right| .
\end{aligned}
$$

The atomic part does, in fact, contain other terms $(N-M$ of them), but these are all decoupled from the cavity and play no further role. Taken together, then, we can write the system Hamiltonian as $H_{S}=h_{0}+\sum_{j=1}^{R_{\Gamma}} h_{j}$, where

$$
\begin{aligned}
h_{j}= & -\Delta_{C}\left|\widetilde{C}_{j}\right\rangle\left\langle\widetilde{C}_{j}\left|-\Delta_{A}\right| \widetilde{A}_{j}\right\rangle\left\langle\widetilde{A}_{j}\right| \\
& +\frac{1}{2} \lambda_{j}\left(\left|\widetilde{C}_{j}\right\rangle\left\langle\widetilde{A}_{j}|+| \widetilde{A}_{j}\right\rangle\left\langle\widetilde{C}_{j}\right|\right)
\end{aligned}
$$

describes the coupling between the $j$ th collective atom and cavity modes, and

$$
h_{0}=\sum_{j=R_{\Gamma}+1}^{M}-\Delta_{C}\left|\widetilde{C}_{j}\right\rangle\left\langle\widetilde{C}_{j}\left|-\Delta_{A}\right| \widetilde{A}_{j}\right\rangle\left\langle\widetilde{A}_{j}\right|
$$

describes the remaining uncoupled elements that arise from a rank-deficient $\Gamma$ matrix. In an obvious matrix representation, the coupled Hamiltonian can be written

$$
h_{j}=\left(\begin{array}{cc}
-\Delta_{C} & \frac{1}{2} \lambda_{j} \\
\frac{1}{2} \lambda_{j} & -\Delta_{A}
\end{array}\right) .
$$

Thus, in the weak excitation limit, we see that we obtain a set of $R_{\Gamma}$ independent two-level coupled atom-cavity systems. These two-level systems have identical detunings, but each has its own effective coupling strength, $\lambda_{j}$. On resonance, $\Delta_{A}=\Delta_{C}=0$, the splitting of the two states in the $j$ th system is simply $\lambda_{j}$.

In the collective basis the driving term reads

$$
H_{D}=\sum_{j=1}^{M}\left(\tilde{\eta}_{j}\left|\widetilde{C}_{j}\right\rangle\left\langle 0\left|+\tilde{\eta}_{j}^{*}\right| 0\right\rangle\left\langle\widetilde{C}_{j}\right|\right)
$$

with the new driving amplitudes $\tilde{\eta}_{j}=\sum_{k=1}^{M} U_{j k}^{*} \eta_{k}$. Thus we see that, since the SVD mixes all cavity modes together (for a generic coupling matrix $G$ ), pumping just one of the original modes pumps all the collective modes.

\section{Effective coupling strengths and the $\Gamma$ matrix}

The cavity transmission spectrum is determined by the eigenvalues $\lambda_{j}$ of the matrix $\Gamma=\sqrt{G G^{\dagger}}$. The elements of $G$ are the microscopic atom-cavity coupling constants $g_{k l}$, which depend on the atomic position: $g_{k l}=g_{k}\left(\mathbf{r}_{l}\right)$. The eigenvalues $\lambda_{j}$, and thus the cavity transmission spectrum, are therefore expected to depend upon the distribution of the atoms within cavity. In the continuum limit, the atomic positions may be described by the continuous density distribution $\rho_{A}(\mathbf{r})$. The matrix product in $G G^{\dagger}$ is then transformed into an average over this atomic distribution:

$$
\left(G G^{\dagger}\right)_{k k^{\prime}} \rightarrow \int d^{3} \mathbf{r} \rho_{A}(\mathbf{r}) g_{k}(\mathbf{r}) g_{k^{\prime}}^{*}(\mathbf{r}) \equiv\left(\left\langle G G^{\dagger}\right\rangle_{A}\right)_{k k^{\prime}}
$$


We describe the cavity modes with the set of HermiteGaussian polynomials $u_{n m}$, which are orthogonal and normalized so as to have a fixed volume integral equal to the volume of the 00 mode $V_{00}=L \pi w_{00}^{2} / 4$, with $L$ being the cavity length and $w_{00}$ being the waist of the 00 mode. Replacing the single mode index $k$ with the double index $(\mathrm{nm})$ of the Hermite-Gaussian polynomials, the coupling constant of the $n m$ mode can be written as

$$
g_{(n m)}(\mathbf{r})=\sqrt{\frac{\mu^{2} \omega_{C}}{2 \hbar \epsilon_{0} V_{00}}} u_{n m}(\mathbf{r}),
$$

where $\mu$ is the atomic dipole moment, $\omega_{C}$ is the cavity resonance frequency, and $\epsilon_{0}$ is the vacuum permittivity. The matrix $G G^{\dagger}$ can then be written as

$$
\begin{aligned}
& \left\langle G G^{\dagger}\right\rangle_{A} \\
& =\frac{\mu^{2} \omega_{C}}{2 \hbar \epsilon_{0} V_{00}}\left(\begin{array}{cccc}
\left\langle u_{00}^{2}\right\rangle_{A} & \left\langle u_{00} u_{11}\right\rangle_{A} & \left\langle u_{00} u_{20}\right\rangle_{A} & \ldots . \\
\left\langle u_{11} u_{00}\right\rangle_{A} & \left\langle u_{11}^{2}\right\rangle_{A} & \langle\ldots\rangle_{A} & \ldots . \\
\ldots & \ldots & \left\langle u_{20}^{2}\right\rangle_{A} & \ldots . \\
\ldots & \ldots & \ldots & \ldots . .
\end{array}\right) .
\end{aligned}
$$

In the limiting cases of uniform and delta-peaked atomic distributions, it is possible to perform the average over atomic degrees of freedom explicitly and find analytic expressions for the effective coupling strengths $\lambda_{j}$, as we now discuss.

\section{Uniform atomic distribution}

We consider first the case of a uniform atomic distribution:

$$
\rho_{A}=\frac{N}{V}
$$

The matrix $\left\langle G G^{\dagger}\right\rangle_{A}$ becomes diagonal due to the orthogonality of the Hermite-Gaussian polynomials:

$$
\left(\left\langle G G^{\dagger}\right\rangle_{A}\right)_{k k^{\prime}}=\frac{\mu^{2} \omega_{C}}{2 \hbar \epsilon_{0} V_{00}} \rho_{A} V_{00} \delta_{k k^{\prime}}=\frac{\mu^{2} \omega_{C}}{2 \hbar \epsilon_{0}} \rho_{A} \delta_{k k^{\prime}}
$$

The eigenvalues of the matrix $\Gamma$ are then degenerate, with the value

$$
\lambda_{k}=\sqrt{\frac{\mu^{2} \omega_{C}}{2 \hbar \epsilon_{0}} \rho_{A}},
$$

and the matrix $\Gamma$ is of full rank, $R_{\Gamma}=M$. The effective couplings $\lambda_{k}$ do not show any dependence on the number of modes $M$, and thus, with a uniform atomic distribution, the multiple degenerate cavity modes do not lead to an enhancement of the coupling. Rather, there exist $M$ such split systems with the same coupling. It should be noted that the cavity states involved in each of these splittings are mixtures of all of the original modes [see Eq. (15)].

\section{Delta-peaked atomic distributions}

We consider now the opposite case of an atomic distribution with all atoms localized in the center of the cavity, $\mathbf{r}=\mathbf{0}$, and we take the density function to be a delta function:

$$
\rho_{A}(\mathbf{r})=N \delta(\mathbf{r}) .
$$

The matrix $G G^{\dagger}$ is readily calculated as

$$
\left\langle G G^{\dagger}\right\rangle_{A}=N\left(\begin{array}{cccc}
g_{(00)}^{2} & 0 & g_{(00)} g_{(22)} & \ldots . \\
0 & 0 & 0 & \ldots . \\
g_{(00)} g_{(20)} & \ldots & g_{(20)}^{2} & \ldots . . \\
\ldots & \ldots & \ldots & \ldots . .
\end{array}\right),
$$

where all the coupling constants $g_{(\mathrm{nm})}$ are evaluated at $\mathbf{r}=\mathbf{0}$. The matrix $G G^{\dagger}$ has a single nonzero eigenvalue $\left(\lambda_{1}^{2}\right.$, say) given by

$$
\lambda_{1}^{2}=N \sum_{k=1}^{M} g_{k}
$$

and all the others equal to zero $\left(R_{\Gamma}=1\right)$. In this case the presence of $M$ degenerate modes leads to an enhancement of the atom-cavity coupling, in contrast to the case analyzed previously. For the case $g_{k} \sim g_{00}$, we recover the known result [24] $\lambda_{1} \sim g_{00} \sqrt{N M}$ such that the single effective coupling scales with both the square root of the number of both atoms and cavity modes.

That $\Gamma$ is rank deficient in this case implies that the cavity transmission will display a peak for zero detuning, provided that any of the decoupled collective modes (i.e., those corresponding to a zero eigenvalue) are pumped. This, as well as the case of more generic atomic distributions, will be further analyzed with the help of numerical simulations.

\section{E. Quantum master equation}

To include the effects of cavity losses and spontaneous emission we study the Liouville-von-Neumann equation for the atom-cavity density matrix $\rho$, which reads

$$
\frac{d}{d t} \rho=-i[H, \rho]+\mathcal{L}_{\text {loss }}[\rho]+\mathcal{L}_{\text {spon }}[\rho] .
$$

Here

$$
\mathcal{L}_{\text {loss }}[\rho]=\frac{1}{2} \sum_{m=1}^{M} \kappa_{m}\left\{2 a_{m} \rho a_{m}^{\dagger}-a_{m}^{\dagger} a_{m} \rho-\rho a_{m}^{\dagger} a_{m}\right\}
$$

describes the loss at rate $\kappa_{m}$ from cavity mode $m$, and

$$
\mathcal{L}_{\text {spon }}[\rho]=\frac{1}{2} \sum_{n=1}^{N} \gamma_{n}\left\{2 \sigma_{n}^{-} \rho \sigma_{n}^{+}-\sigma_{n}^{+}{\sigma_{n}^{-}}^{-} \rho-\rho \sigma_{n}^{+} \sigma_{n}^{-}\right\}
$$

describes the spontaneous emission from the atoms at rate $\gamma$. For simplicity, in calculations we assume that all modes decay at the same rate $\kappa_{m}=\kappa ; \forall m$.

Our simulations follow the dynamical evolution of $N$ twolevel atoms and $M$ cavity modes where the atoms are randomly spatially distributed and the modes are Hermite-Gaussian $\mathrm{TEM}_{n m}$ modes. By simulating single trajectories of the $N$ atom and $M$ mode system, we are able to study the dynamics of a cavity with four modes and containing up to $1.6 \times 10^{4}$ atoms. The experimentally observed four strongest modes were considered in our simulations. The atom-cavity couplings $g_{k a}$ are determined by assuming an atomic distribution within the cavity corresponding to the experimental one. We generate $N$ random positions according to the atomic distribution and then calculate the $N \times M$ couplings for the $N$ atoms coupled to the $M$ modes. Pumping only one mode $\left(\mathrm{TEM}_{00}\right)$, the evolution of 
the photon number in each mode, $\left|\alpha_{n m}\right|^{2}$, shows the coupling between modes induced via the atom-cavity interaction.

Our simulation deals with a number of atoms smaller than the one considered experimentally and a larger single-atom coupling constant for computational simplicity. We verified numerically that the same results produced for a small number of atoms and a large single-atom coupling apply for a large number of atoms and a weak single-atom coupling, provided that the system is in the collective strong-coupling regime.

\section{Mode-mode coupling}

We first consider an atomic distribution closely representing the experimental one and investigate the coupling between the modes mediated by the atomic sample. Our numerical results for the steady-state intracavity photon number for the different modes are reported in Fig. 4. These results show that there is

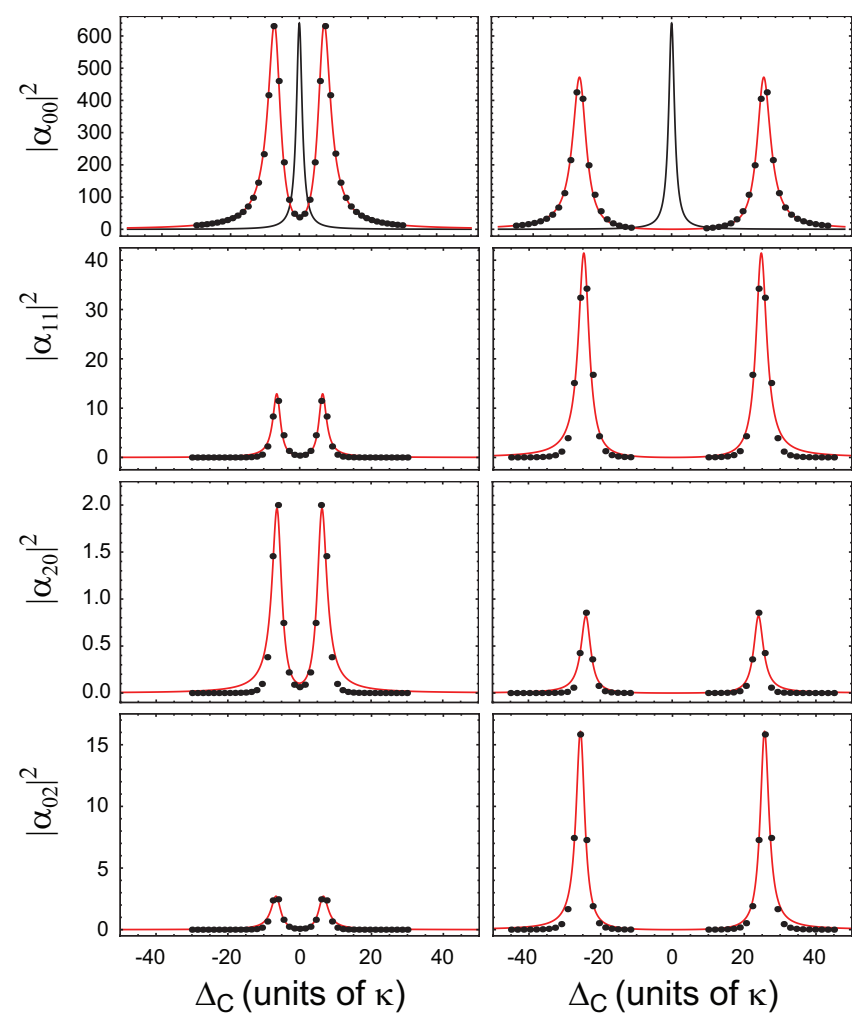

FIG. 4. (Color online) Numerical results for the intracavity photon number as a function of $\Delta_{C}$ for the the case of 1000 (left) and 16000 (right) atoms Gaussian distributed in a realistic threedimensional (3D) multimode cavity field with the available TEM modes 00, 20, 02, and 11. The (black) dots are the actual numerical results from the simulation, while the solid (red) lines are the best fits with Eq. (2). Dashed (black) lines are results for an empty cavity, reported for comparison. The parameters of the calculation are as follows. The maximum value for the coupling constant for the 00 mode is $g=\kappa$, with the individual couplings for the different atoms calculated depending on their position (see text). $\gamma=3.25 \kappa$. The mode pumped is the $\mathrm{TEM}_{00}$ mode with $\eta_{00}=0.1 \kappa$, and the displayed steady-state photon number is multiplied by $10^{6}$. The atomic distribution is similar to the experiment following a cloud with Gaussian width $\sigma_{z}=1.25 w_{0}$ and $\sigma_{r}=1.6 \sigma_{z}$, where $w_{0}$ is the waist of the cavity field and $z$ is perpendicular to the cavity axis. an effective atom-mediated coupling between the modes: by pumping only one mode of the cavity (mode 1 ), the presence of the atoms leads to scattering also into the other modes, and a cavity field is built up also for the modes not pumped by the laser. This is in agreement with our experimental results.

We notice that in general we would expect to see a multipeaked mode profile for each cavity optical mode with splittings corresponding to the different eigenvalues of the $\Gamma$ matrix. However, both experiment and numerical simulations show just two peaks in the transmission spectrum of each mode and no features at resonance with the bare cavity frequency.

The observed two-peaked structure can be understood by studying the eigenvalues of the $\Gamma$ matrix as derived from the atomic density function. In the limit of a spatially uniform atom distribution the $\Gamma$ matrix becomes diagonal and its eigenvalues become degenerate, consistent with the observed two-peak structure.

In our system the atomic distribution is not exactly uniform but large enough to make the effective couplings $\lambda_{k}$ quasidegenerate, hence the absence of a multipeaked structure in the cavity transmission spectrum for the different modes.

\section{Line broadening}

While the quasidegeneracy of the effective couplings does not allow the resolution of a multipeak structure, it leads to a broadening of the cavity transmission peaks with increasing number of atoms. As the eigenvalues scale with $N^{1 / 2}$, the cavity transmission peaks should broaden with the same scaling. We run numerical simulations to analyze the width of the cavity transmission spectrum as a function of the number of atoms in the cavity. In our calculations we selected the parameters corresponding to our experiment, with results

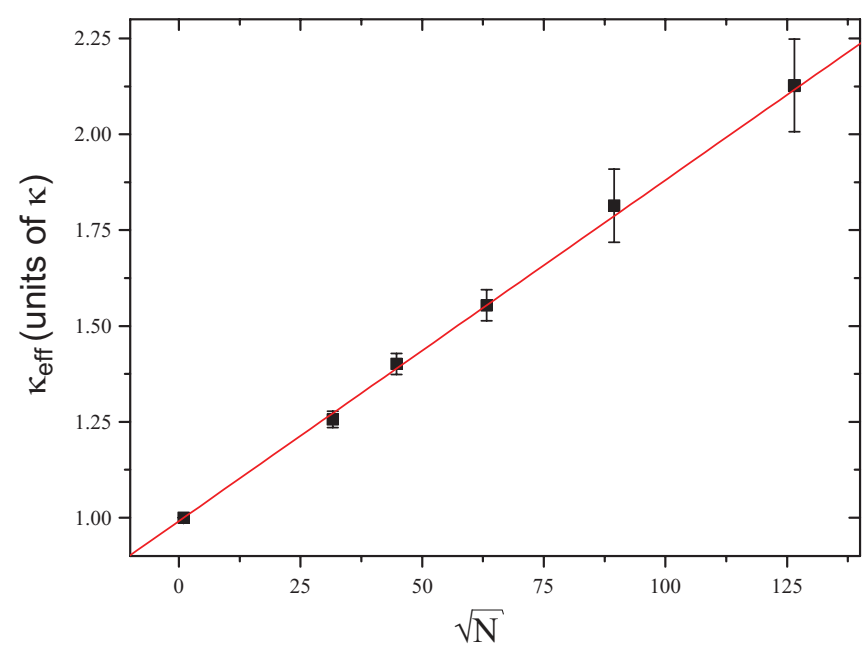

FIG. 5. (Color online) Value for the parameter $\kappa_{\text {eff }}$ as derived from fitting the numerically simulated transmission data of the $\mathrm{TEM}_{00}$ mode data with Eq. (2) as a function of the number of atoms $N$. The atoms are randomly distributed in a 3D Gaussian centered with the center of the cavity and with width $\sigma_{x}=1.3 w_{0}$ and $\sigma_{y}=\sigma_{z}=1.6 \sigma_{x}$. The cavity has four transverse modes: $00,11,20$, and 02 . The mode pumped is the 00 mode with $\eta_{00}=0.1 \kappa$. Since the eigenvalues of the system are quasidegenerate the resulting effective $\kappa$ scales linearly with $\sqrt{N}$, where $N$ is the total atom number in the distribution. 


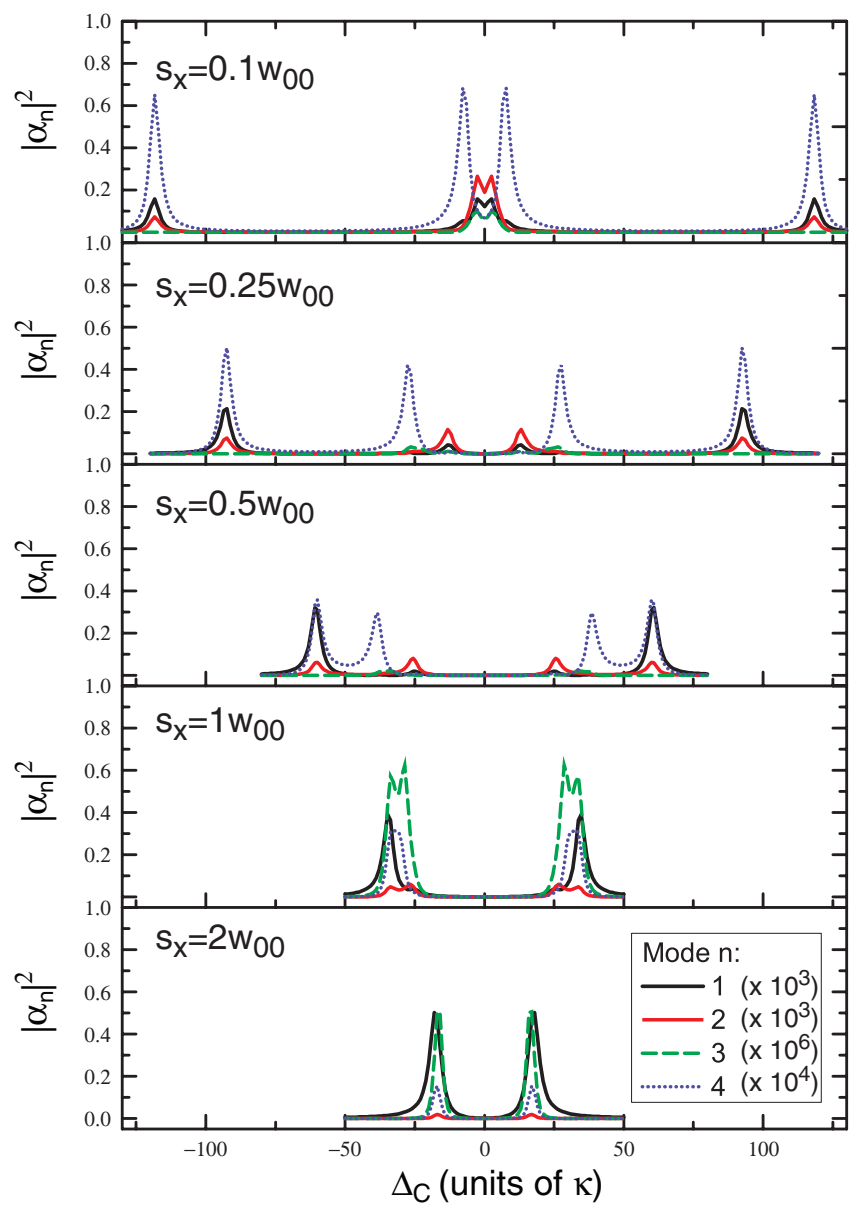

FIG. 6. (Color online) Numerical simulations for the cavity transmission spectroscopy for different spatial extensions of the atomic cloud. All the data sets are for a sample of 16000 atoms and a cavity with four degenerate modes. The atoms are 3D Gaussian distributed with width $\left(s_{x}, 2 s_{x}, 2 s_{x}\right)$, expressed in the figure in terms of the fundamental Gaussian cavity mode waist $w_{00}$. The different data plots refer to different widths $s_{x}$ of the atomic distribution. The mode pumped is the 00 mode with $\eta_{00}=0.1 \kappa$.

shown in Fig. 5. The simulations show the broadening of the width of the resonance with the dependence $N^{1 / 2}$ as observed in our experiment. This can be traced back to quasidegenerate eigenvalues $\lambda_{j}$ which scale as $N^{1 / 2}$, thus leading to a broadening of the transmission peaks with the same dependence on the atom number. That is, the transmission peak is actually made up of the several near degenerate modes; i.e., the experimentally observed line broadening is actually an overlap of several lines which are each too broad to be resolved. This behavior holds as long as the spreading $\Delta \lambda$ of the eigenvalues is much smaller than the empty cavity linewidth $\kappa(0)$. Otherwise the spectrum can separate in several peaks.

\section{Multipeaked cavity transmission}

We generalize our analysis by considering atomic distributions different from the one corresponding to our experiment. This allows us to explore multipeaked cavity transmission profiles.

Our simulations examine the structure of the cavity transmission spectrum for different atomic distributions, with results as those shown in Fig. 6. In all our simulations, we pump only the fundamental mode (mode 00).

For a broad atomic distribution (bottom panel), only two peaks, symmetrically displaced with respect to the origin, are present in the mode spectrum. This is consistent with our analytic results for a uniform distribution. For narrower atomic distributions the mode structure is more complicated, in agreement with the fact that the eigenvalues $\lambda_{j}$ are not degenerate any more. Finally, for atomic distributions more and more localized in the middle of the cavity, the mode structure simplifies again: a central peak appears, together with two symmetrically displaced resonances. This is in agreement with our analytic results for a delta-peaked atomic distribution.

\section{CONCLUSIONS}

In conclusion, in this work we studied an atom-cavity system, in which the cavity has several degenerate transverse modes. This was obtained by using a nearly confocal cavity. Experiments were performed in the collective strong-coupling regime. Mode-resolved cavity transmission spectroscopy revealed well-resolved atom-cavity resonances for several cavity modes. In agreement with our theoretical model, the experiment shows that the cavity modes are coupled via the atomic ensemble contained in the cavity.

The present work is of relevance to a number of applications, from cavity cooling of atoms and molecules, where the use of nearly confocal cavities is predicted to enhance the cooling, to cavity quantum information processing, where atom-mediated interaction between degenerate modes may allow novel implementation of quantum gates as well as the production of highly entangled states of different cavity mode states. Finally, our results are also relevant to the experimental realization of quantum spin glasses with ultracold atoms.

\section{ACKNOWLEDGMENTS}

This work is supported by the Engineering and Physical Sciences Research Council (Grant No. EP/H049231/1) and the Royal Society.
[1] Cavity Quantum Electrodynamics, edited by P. Berman (Academic, Boston, 1994).

[2] H. Mabuchi and A. C. Doherty, Science 298, 1372 (2002).

[3] J. M. Raimond, M. Brune, and S. Haroche, Rev. Mod. Phys. 73, 565 (2001)
[4] M. Brune, E. Hagley, J. Dreyer, X. Maître, A. Maali, C. Wunderlich, J. M. Raimond, and S. Haroche, Phys. Rev. Lett. 77, 4887 (1996).

[5] T. Pellizzari, S. A. Gardiner, J. I. Cirac, and P. Zoller, Phys. Rev. Lett. 75, 3788 (1995). 
[6] S. J. van Enk, J. I. Cirac, and P. Zoller, Phys. Rev. Lett. 79, 5178 (1997).

[7] J. Pachos and H. Walther, Phys. Rev. Lett. 89, 187903 (2002).

[8] L. M. Duan, A. Kuzmich, and H. J. Kimble, Phys. Rev. A 67, 032305 (2003).

[9] Q. A. Turchette, C. J. Hood, W. Lange, H. Mabuchi, and H. J. Kimble, Phys. Rev. Lett. 75, 4710 (1995).

[10] P. Horak, G. Hechenblaikner, K. M. Gheri, H. Stecher, and H. Ritsch, Phys. Rev. Lett. 79, 4974 (1997).

[11] V. Vuletic and S. Chu, Phys. Rev. Lett. 84, 3787 (2000).

[12] P. Domokos and H. Ritsch, J. Opt. Soc. Am. B 20, 1098 (2003).

[13] M. G. Raizen, R. J. Thompson, R. J. Brecha, H. J. Kimble, and H. J. Carmichael, Phys. Rev. Lett. 63, 240 (1989); A. K. Tuchman, R. Long, G. Vrijsen, J. Boudet, J. Lee, and M. A. Kasevich, Phys. Rev. A 74, 053821 (2006).

[14] Y. Colombe, T. Steinmetz, G. Dubois, F. Linke, D. Hunger, and J. Reichel, Nature (London) 450, 272 (2007); F. Brennecke, T. Donner, S. Ritter, T. Bourdel, M. Köhl, and T. Esslinger, ibid. 450, 268 (2007).
[15] T. Taneichi and T. Kobayashi, Chem. Phys. Lett. 378, 576 (2003).

[16] V. Vuletić, H. W. Chan, and A. T. Black, Phys. Rev. A 64, 033405 (2001).

[17] F. O. Prado, F. S. Luiz, J. M. Villas-Bôas, A. M. Alcalde, E. I. Duzzioni, and L. Sanz, Phys. Rev. A 84, 053839 (2011); Y. Dong, X. Zou, S. Zhang, S. Yang, C. Li, and G. Guo, J. Mod. Opt. 56, 1230 (2009).

[18] P. Strack and S. Sachdev, Phys. Rev. Lett. 107, 277202 (2011).

[19] M. L. Terraciano, R. Olson Knell, D. G. Norris, J. Jing, A. Fernández, and L. A. Orozco, Nat. Phys. 5, 480 (2009).

[20] A. Wickenbrock, P. Phoonthong, and F. Renzoni, J. Mod. Opt. 58, 1310 (2011).

[21] R. J. Thompson, G. Rempe, and H. J. Kimble, Phys. Rev. Lett. 68, 1132 (1992).

[22] E. T. Jaynes and F. W. Cummings, Proc. IEEE 51, 89 (2005).

[23] M. Tavis and F. W. Cummings, Phys. Rev. 170, 379 (1968).

[24] G. J. Papadopoulos, Phys. Rev. A 37, 2482 (1988). 